\title{
Editorial: Materials Research in the Physical Review Journals
}

In February 2017 the American Physical Society (APS) launched Physical Review Materials (PRMaterials), with the aim of giving our authors more choice in communicating with the increasingly diverse audience for materials research. The journal also intends to broaden the footprint of materials research covered by the Physical Review journals to include a previously underserved multidisciplinary community. This community includes not only physicists but also materials scientists, chemists, and engineers. The initial response from the research community has been very positive, both in terms of quality and quantity of manuscripts submitted, and PRMaterials has made a strong debut.

It now seems timely to reiterate the focus of each journal in the main Physical Review series that publishes papers related to materials research. Our intention in doing so is to furnish prospective authors with the most up-to-date information, so that they can select the journal that is most appropriate for their work.

Accordingly, when considering to which journal a materials paper should be submitted, we would like to remind you of the following:

- Physical Review $B$ focuses on the exploration and understanding of the physical properties and phenomena in materials of relevance to condensed matter physics and allied disciplines.

- Physical Review E publishes papers concerning the physical properties and collective phenomena of soft matter and biomaterials.

- Physical Review Applied features materials papers important for devices and their practical applications.

- Physical Review Materials emphasizes the prediction, discovery, synthesis, characterization, processing, structure, properties, and modeling of materials of interest to the multidisciplinary materials community.

PRMaterials does not replace any of the journals that have traditionally published materialsrelated research, nor does its arrival change their scope. Rather, it expands the scope of the Physical Review family. If, as is often the case, a paper is suitable for more than one journal, we advise authors to submit to the journal that best addresses the target audience for their research. As previously, it is possible to transfer papers between journals, and the placement of individual papers will be informed by the wishes of the authors, along with advice from editors, referees, and editorial board members.

We are pleased to see the new journal in the Physical Review family and to offer a wider choice of venues for the publication of materials research. As has always been the case, the Physical Review journals will remain responsive to the community and will continue to evolve to serve the respective research fields and needs of our readers and authors.

Chris Leighton

Editor, Physical Review Materials

Laurens W. Molenkamp

Editor, Physical Review B

Eli Ben-Naim

Editor, Physical Review E

Stephen Forrest

Published 23 August 2017

Editor, Physical Review Applied

DOI: 10.1103/PhysRevApplied.8.020001 